\title{
Premières observations sur le comportement d'un panneau de paroi moulée instrumenté au nouveau port extérieur du Havre
}

\author{
Sonja Marten ${ }^{(a)}$ et Luc Delattre ${ }^{(a)}$, \\ Joseph Lavisse $^{(\mathrm{b})}$ et Jean-Luc Gobert ${ }^{(\mathrm{b})}$, Philippe Joignant ${ }^{(\mathrm{c})}$ \\ (a)Doctorante ,Docteur; Laboratoire Central des Ponts et Chaussées, 58 Bd Lefebvre, \\ 75015 Paris,prenom.nom@lcpc.fr \\ (b)Ingénieurs, Solétanche-Bachy, 6 rue de Watford, 92005 Nanterre Cedex, \\ prenom.nom@soletanche-bachy.com \\ (c)Ingénieur, Port Autonome du Havre, Terre-plein de la Barre, BP 1413, \\ 76067 Le Havre Cedex, prenom.nom@havre-port.fr
}

\section{Résumé}

Le nouveau mur de quai de «Port 2000 », situé dans l'estuaire de la Seine au Havre (France), est actuellement en cours de construction. Le LCPC, en coopération avec le Port Autonome du Havre, l'entreprise Solétanche-Bachy et le CETE Normandie-Centre, a instrumenté un panneau de paroi moulée de ce quai. L'étude est destinée à approfondir la compréhension du comportement de tels ouvrages portuaires, et de recueillir des données en vue d'améliorer les méthodes de calcul.

\begin{abstract}
$\underline{\text { Abstract }}$
The new quay wall "Port 2000", located in the estuary of the Seine River in Le Havre (France), is currently under construction. In cooperation with the Port of Le Havre Authority, the contractor Solétanche-Bachy and the CETE Normandy-Centre, the LCPC has undertaken a monitoring and evaluation for this project. The case study of Port 2000 is designed to improve knowledge about the behaviour of such harbour structures and to create a database that will be useful to improve calculation methods.
\end{abstract}

Mots-clés: mur de quai, ouvrage portuaire, paroi moulée, instrumentation

Keywords: quay wall, harbour structure, diaphragm wall, instrumentation

\section{Introduction}

La croissance du volume de marchandises conteneurisées et la construction de navires à porte-conteneurs de plus en plus grands avec un tirant d'eau croissant ainsi que des temps d'escale réduits nécessite que les ports adaptent leurs moyens d'accueil et de stockage pour accroître leur rôle sur le marché européen. Dans ce contexte, le Port du Havre, premier port français pour les trafics conteneurisés ${ }^{1}$, équipe ses quais existants de nouveaux portiques et augmente sa capacité en construisant à l'extérieur du port existant, en première phase, un nouveau quai en eau profonde de $1400 \mathrm{~m}$ utiles de long directement accessible à toute heure de la marée.

L'ouvrage comprend non seulement le mur de quai lui-même, mais aussi un système d'endiguement et de nouveaux terre-pleins entre l'ancienne digue et le futur chenal (figure 1). La section instrumentée de l'ouvrage se trouve dans l'extrémité Ouest du quai. Les résultats de cette étude vont servir à compléter les observations faites sur d'autres constructions comparables, notamment le quai d'Osaka ${ }^{2}$, situé aussi au port du Havre. 


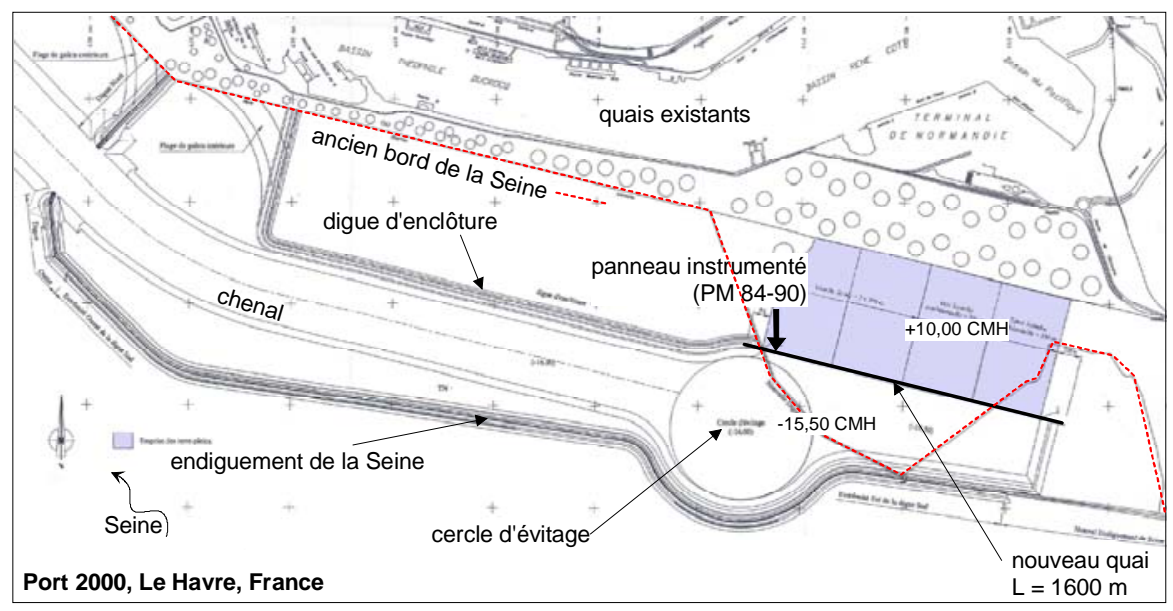

Figure 1. Vue en plan du site du nouveau port.

\section{Présentation du site}

Les altitudes du projet sont rapportées au zéro des cartes marines du Havre (CMH) lequel est situé 4,38 m au-dessous du zéro du nivellement général de la France. Le site a été gagné sur la Seine par remblaiement hydraulique de matériaux sablo-limoneux jusqu'à la cote +10 $\mathrm{CMH}$ environ. Sous-jacent aux remblais, l'ancien lit du fleuve se trouvait initialement vers la cote $0 \mathrm{CMH}$. Il est constitué de dépôts récents alluvionnaires ou dépôts vasards.

Ces deux couches sont entièrement terrassées en avant du quai lors des opérations de dragage à la cote nominale, et remplacées en arrière après la pose des tirants.

Sous ces dépôts, la formation alluviale principale, d'une épaisseur de 19 à $29 \mathrm{~m}$, est composée de sables fins à grossiers, répartis en deux couches principales: en partie supérieure, des sables fins coquilliers, généralement assez propres et pulvérulents, localement finement stratifiés par des lits silteux; en partie inférieure, des sables fins à grossiers gris-vert avec graviers roulés et galets et des inclusions de sables fins à moyens. En profondeur, des sédiments fins continentaux, constitués de silts fins plastiques, constituent un horizon très peu perméable. Sous les sédiments fins plastiques est disposée la couche de "graves de fond » constituée de sables grossiers, de graviers assez anguleux, de galets de silex et de blocs de plus de $100 \mathrm{~mm}$. Enfin, le substratum est formé par des argiles de Villerville (marnes noires fermes à raides). Il est situé entre -29 et $-32 \mathrm{CMH}$. Le tableau suivant résume les caractéristiques mécaniques tirées d'essais in situ et en laboratoire.

Tableau 1. Synthèse des caractéristiques géotechniques du sol (profil à proximité du panneau instrumenté)

\begin{tabular}{lccccccc}
\hline \hline nature des terrains & $\begin{array}{c}\text { cotes CMH } \\
\mathrm{m}\end{array}$ & $\begin{array}{c}\gamma \\
\mathrm{kN} / \mathrm{m}^{3}\end{array}$ & $\begin{array}{c}\varphi^{\prime}\left(\varphi_{\mathrm{u}}\right) \\
\text { degré }\end{array}$ & $\begin{array}{c}\mathrm{c}^{\prime}\left(\mathrm{c}_{\mathrm{u}}\right) \\
\mathrm{kPa}\end{array}$ & $\begin{array}{c}\mathrm{q}_{\mathrm{c}} \\
\mathrm{MPa}\end{array}$ & $\begin{array}{c}\mathrm{p}_{\mathrm{L}}-\mathrm{p}_{0} \\
\mathrm{MPa}\end{array}$ & $\begin{array}{c}\mathrm{E}_{\mathrm{M}} \\
\mathrm{MPa}\end{array}$ \\
\hline remblais nouveaux & $+10,2$ à $-7,5$ & 19,5 & 35 & 0 & 8 & & \\
remblais hydrauliques & $\mathrm{TN}$ à $+0,0$ & 18,0 & 33 & 0 & 10 & 0,7 & 7 \\
dépôts organiques récents & $+0,0$ à $-2,0$ & 16,0 & $20(0)$ & $0(2$ à 15$)$ & 1 & & \\
sables supérieurs & $-2,0$ à $-5,0$ & 19,0 & 37 & 0 & 15 & 2,3 & 27 \\
sables inférieurs & $-5,0$ à $-8,0$ & 17,0 & 29 & 0 & 3 & 0,9 & 6 \\
sables inf. denses & $-8,0$ à -17,0 & 20,0 & 35 & 0 & 10 & 1,7 & 16 \\
sables très denses & $-17,0$ à $-24,8$ & 22,5 & 39 & 0 & 30 & 4,2 & 46 \\
silts inférieurs & $-24,8$ à $-26,5$ & 17,0 & $29(0)$ & $0(100)$ & - & 1,5 & 22 \\
graves de fond & $-26,5$ à -29,5 & 22,0 & 40 & 0 & - & 4,6 & 36 \\
argiles de Villerville & substratum & 20,0 & $20(0)$ & $20(200)$ & - & 1,9 & 22 \\
\hline \hline
\end{tabular}


Sur le plan hydrologique, trois nappes différentes ont été identifiées :

-la nappe superficielle, située au-dessus des dépôts organiques récents. Son toit varie entre $+10,00 \mathrm{CMH}$ à l'Est et +6,60 CMH au Nord-Ouest. Elle n'existe plus après terrassement ; -la nappe des sables s'établit à la cote $+6,50 \mathrm{CMH}$ environ ;

-la nappe des graves suit la marée avec une amplitude amortie, mais sans décalage temporel mesuré par les piézomètres.

La marée varie entre $+0,10 \mathrm{CMH}$ et $+9,10 \mathrm{CMH}$. Les terrassements de part et d'autre de la paroi se font à l'abri d'un rabattement prévu à $-1,00$ et $-8,50 \mathrm{CMH}$ respectivement. Aux extrémités du quai, compte tenu des débits à pomper, il a été réalisé un voile d'étanchéité ancré dans les argiles. Côté Seine, le voile se trouve entre 22 et $35 \mathrm{~m}$ devant la paroi ; côté terre-plein, le talus de déblai pour la mise en place des tirants a conduit à placer le voile à 90 $\mathrm{m}$ derrière la paroi moulée.

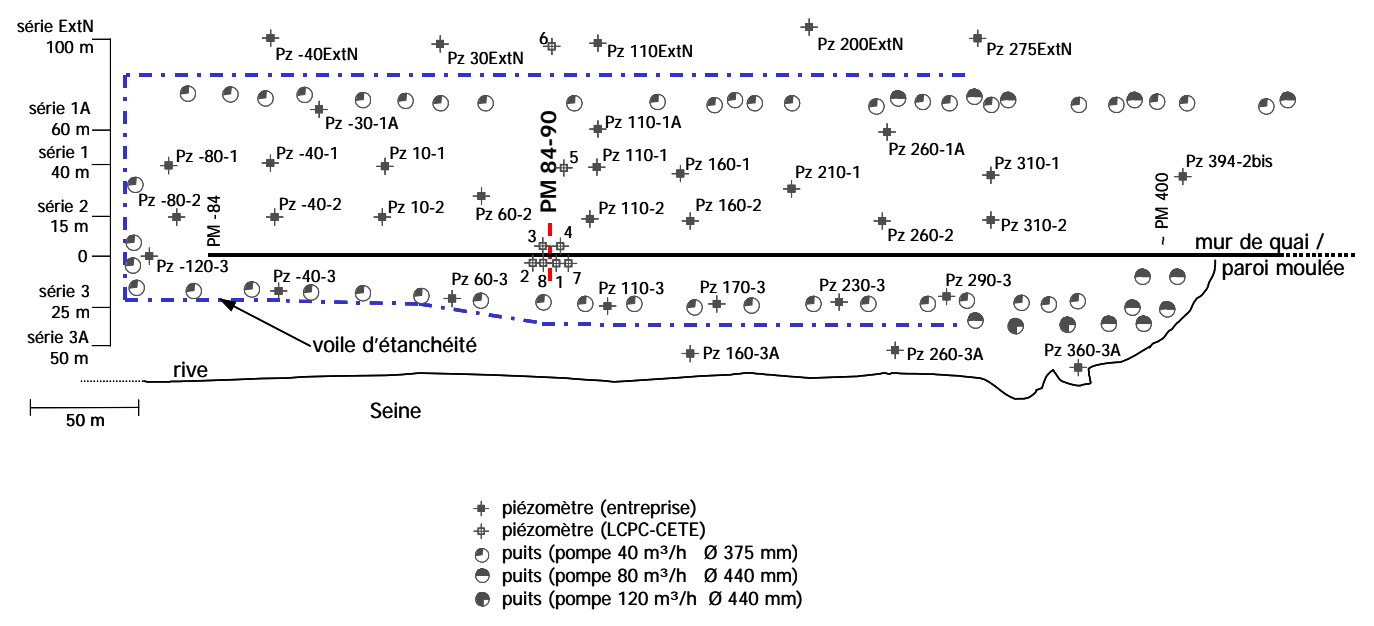

Figure 2. Vue en plan de la partie Ouest du quai. Paroi étanche, dispositif de rabattement et piézomètres

\section{Présentation de l'ouvrage}

L'ouvrage est constitué par une paroi moulée d'une épaisseur de 1,20 $\mathrm{m}$ et d'une profondeur de $40 \mathrm{~m}$ ancrée dans les argiles de Villerville, surmontée par une poutre de couronnement en béton armé de 3,43 m de largeur et de 1,50 m de hauteur. L'ensemble est muni d'un masque d'accostage de 9,10 m de hauteur et 1,60 $\mathrm{m}$ de largeur. La paroi est retenue par deux nappes de tirants passifs faiblement inclinés, mis en place dans les terrains remblayés après terrassement sur $17 \mathrm{~m}$ de hauteur environ derrière la paroi. La figure 3 montre la coupe de la structure dans la zone instrumentée. Les rails du futur portique se trouvent d'une part sur la poutre de couronnement dans l'axe de la paroi moulée, et d'autre part sur une semelle superficielle filante située $35 \mathrm{~m}$ en arrière. 


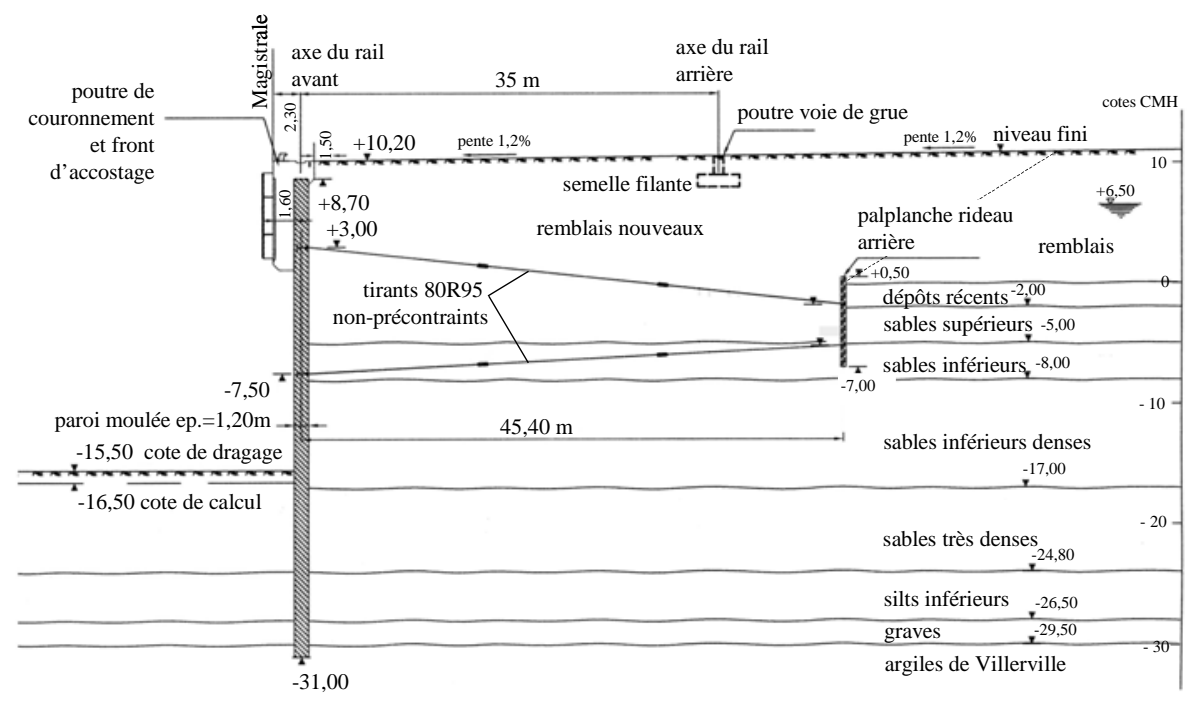

Figure 3. Coupe de la paroi au niveau du panneau instrumenté

\section{Instrumentation mise en place}

L'instrumentation mise en place concerne différents aspects, à savoir :

-la déformation de la paroi suivie par quatre inclinomètres, deux étant limités à la hauteur de la paroi moulée et deux étant prolongés dans le terrain sous-jacent, -la déformation du terrain entre la semelle de fondation du portique et le rideau d'ancrage suivie par 2 inclinomètres,

-la déformation du rideau d'ancrage mesurée par deux inclinomètres,

-le déplacement de la tête de la paroi suivi par des mesures topographiques,

-les efforts dans les tirants mesurés à l'aide de 40 extensomètres à cordes vibrantes et,

-l'évolution des nappes est observée à l'aide d'un ensemble de piézomètres.

La figure 4 montre l'ensemble du dispositif. Les inclinomètres de la paroi ont une profondeur de 49,50 m (encastrés dans les argiles de Villerville) ou de 39,50 m (inclinomètres arrêtés dans la paroi). Dans les remblais récents et sur les rideaux d'ancrage, les inclinomètres sont installés sur une profondeur de $25 \mathrm{~m}$ : leur pied se trouve dans les sables denses. Les piézomètres de grande longueur disposés près de la paroi sont équipés de capteurs de pression et reliés à une centrale d'acquisition munie d'un modem GSM qui permet de faire des mesures à distance. Les deux piézo-mètres de longueur plus réduite supplémentaires ont été installés côté bassin. Les piézomètres mis en oeuvre dans les nouveaux remblais entre la paroi et le rideau, et derrière le voile étanche, sont autonomes avec des stockages de données individuels. Le suivi est complété par les résultats de mesures piézométriques de l'Entreprise (Solétanche-Bachy). 


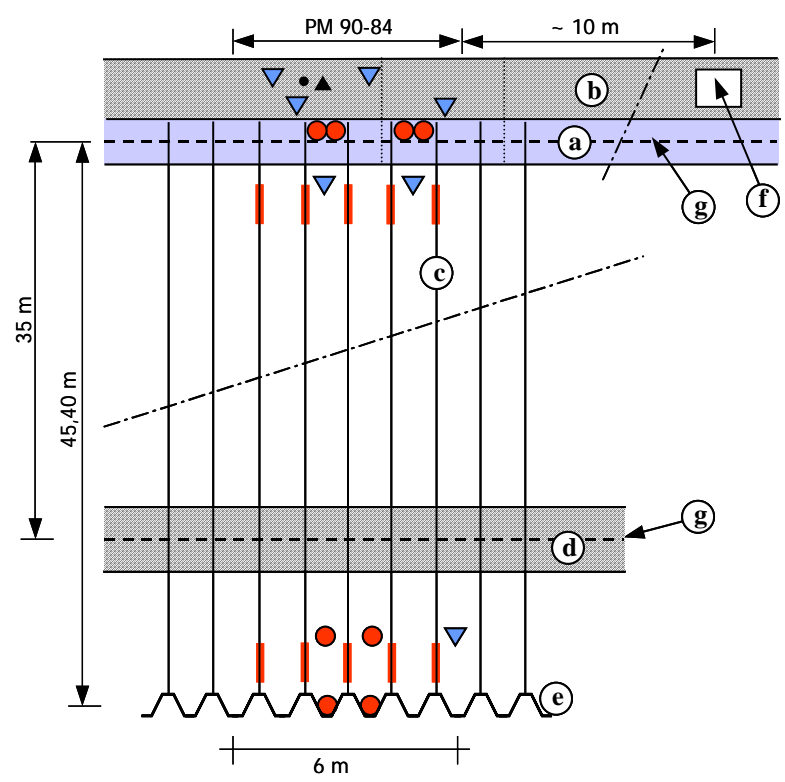

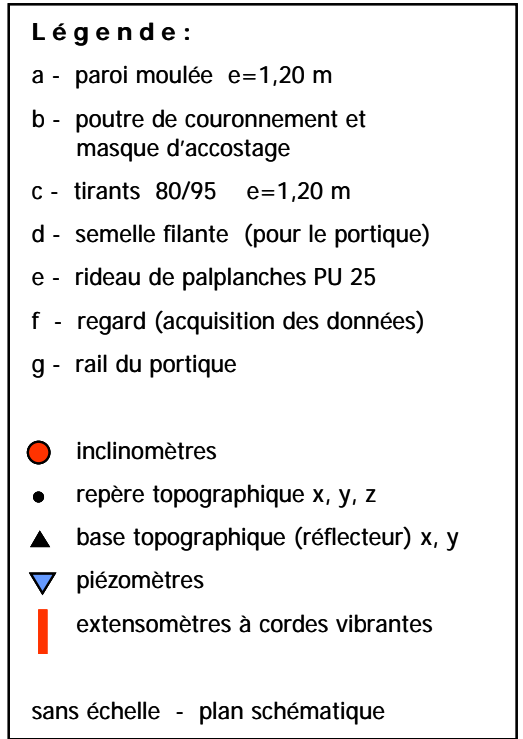

Figure 4. Dispositif d'instrumentation de la paroi et du terrain en arrière.

\section{Phasage des travaux}

Les figures suivantes présentent le phasage des travaux et de l'instrumentation. La phase initiale correspond à la fin de la construction de la paroi et du voile étanche à l'Ouest. Les nappes sont dans leur état initial. Les deux inclinomètres placés dans la paroi et encastrés dans les argiles de Villerville ont été scellés en mars 2003. Les mesures inclinométriques ont débuté en avril 2003.

(0) Phase initiale

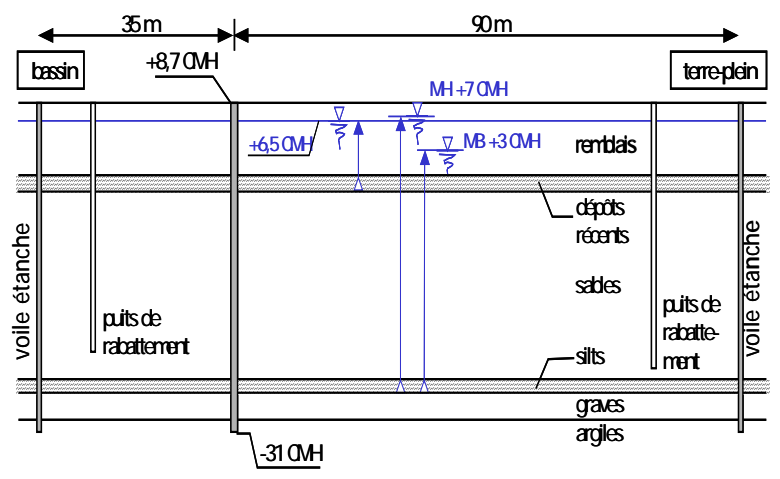

(1) Excavation aval et superstructure

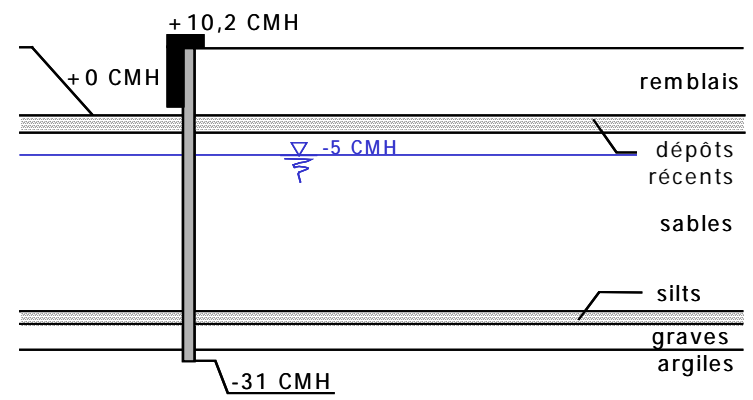


(2) Terrassement amont et tirants

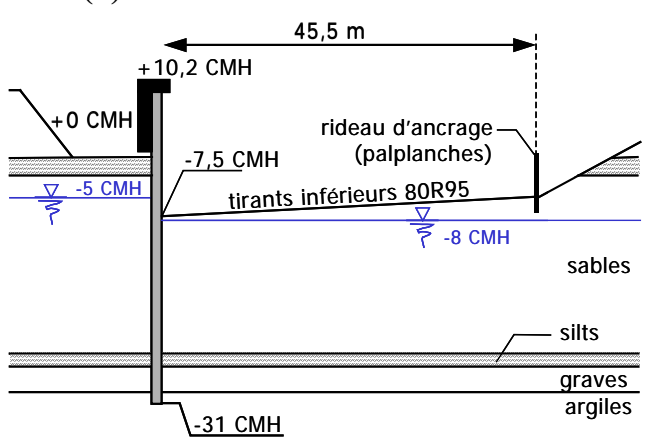

(3) Remblaiement

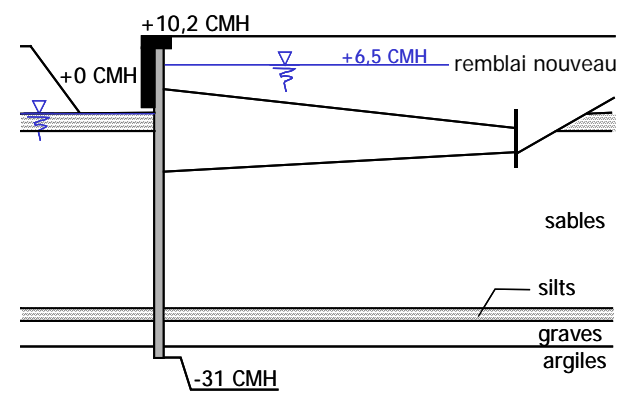

(4) Dragage

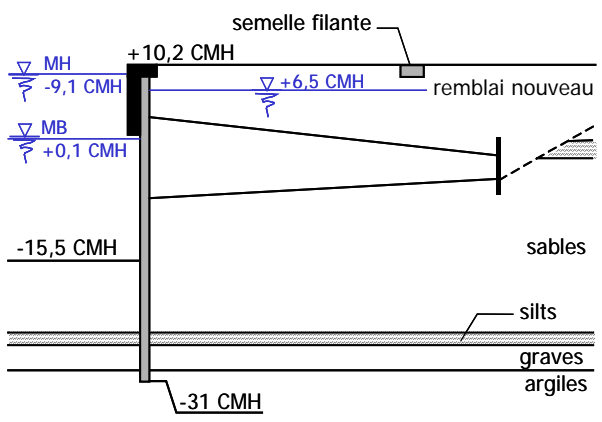

Figure 5. Etapes principaux des travaux

(1) A l'abri du rabattement à -5,00 CMH environ, le terrain est terrassé devant la paroi jusqu'à la cote $+0,00 \mathrm{CMH}$ sur quelques mètres de large.

La superstructure en béton armé est construite en novembre 2003. Le sommet de la poutre de couronnement est à $+10,20 \mathrm{CMH}$, et le bas du masque d'accostage à $+1,10 \mathrm{CMH}$. A partir de cette phase, les équipements du quai sont mis en place.

(2) Après rabattement à $-8 \mathrm{CMH}$ environ derrière la paroi, le terrain est terrassé jusqu'au niveau du lit des tirants inférieurs. Les tirants et le rideau d'ancrage sont mis en place. Deux extensomètres à corde vibrante sont installés à chaque extrémité de 5 tirants. Deux tubes de réservation pour les inclinomètres arrières sont soudés aux pal-planches. En février 2004, le terrassement amont est à $0 \mathrm{CMH}$ environ

(3) Le terrain est remblayé jusqu'à la cote du lit de tirants supérieur, et ces derniers sont mis en place. Comme les tirants inférieurs, cinq des tirants supérieurs sont équipés de deux paires d'extensomètres, une paire à chaque extrémité.

Le rabattement de nappe est diminué au fur et à mesure des remblaiements. Ensuite, le remblai est terminé derrière la paroi et les tubes de réservation des inclinomètres rehaussés jusqu'à la cote $+10 \mathrm{CMH}$. Enfin, deux inclinomètres sont installés au niveau du rideau 
d'ancrage et deux autres disposés dans des forages entre la future voie de grue arrière et le rideau.

(4) Réalisation de la fondation arrière du portique. Le quai est dragué à l'aide d'une drague stationnaire à désagrégateur. La cote de dragage nominale est de -15,5 CMH. A partir de cette phase, l'ouvrage se trouve en contact direct avec la mer. Les digues d'enclôture sont terminées et le quai est mis en service. Tout le dispositif d'instrumen-tation est donc opérationnel et les relevés sont effectués une fois par mois et plus (voir figure 6).

\section{$\underline{\text { 6.Présentation des premiers résultats de mesure }}$}

Au début du mois de février 2004, seule une partie de l'instrumentation est disponible : il s'agit du dispositif inclinométrique dans la paroi et de quatre piézomètres devant la paroi (voir figure 4). Un premier dépouillement des mesures inclinométriques a été fait à l'aide du logiciel CAPITOUL $2000^{2}$. La figure 6 représente l'évolution des déplacements mesurés en tête en fonction de l'avancement des travaux.

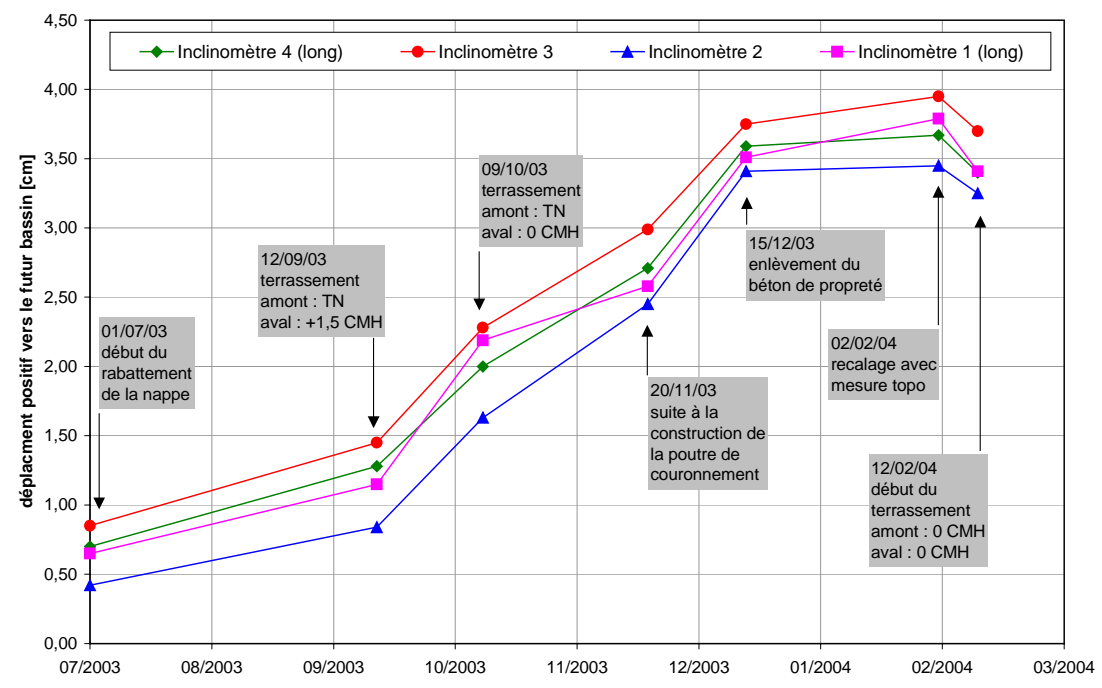

Figure 6. Déplacements cumulés en tête pour les premières phases de construction. (TN : terrain naturel)

Les déplacements augmentent au fur et à mesure de l'avancement du terrassement aval, le fond de fouille étant atteint en octobre 2003. La construction de la poutre de couronnement en mi-novembre conduit à une augmentation des déplacements vers l'aval. Ensuite, l'enlèvement du béton de propreté au-dessous de la poutre d'accostage (qui apparemment représentait un dernier appui de la paroi moulée) mène à une inclinaison supplémentaire. La situation du chantier reste quasiment inchangée jusqu'à début février. Enfin, la mesure du 12/02/2004 montre que le début du terrassement en arrière jusqu' au niveau $0 \mathrm{CMH}$ environ conduit à un léger retrait de 2,8 $\mathrm{mm}$ de la paroi vers l'amont. Les deux mesures topographiques, effectuées le 19/01/04 (mesure initiale) et les 13/02 et 18/02/04, confirment ce recul. Ces premiers résultats inclinométriques indiquent que la structure se déforme sensiblement moins que prévu par les calculs de dimensionnement. Les déplacements cumulés en tête de la paroi montrent un maximum de $3,70 \mathrm{~cm}$ en moyenne lors de la mesure du 02/02/2004. Cette étape de travaux correspond à la première phase de calcul du dimensionnement utilisant la méthode des coefficients de réaction. En considérant les hypothèses fixées au marché incluant des 
sécurités, un déplacement en tête de $9,20 \mathrm{~cm}$ vers le bassin avait été obtenu. Le suivi piézométrique met en évidence que le rabattement de la nappe est d'une intensité plus importante que prévue lors des études préalables. Pour la période de septembre à décembre 2003, côté Seine, les mesures piézométriques (faites à marée haute approximativement) varient entre les cotes $-8,00$ et $-3,00 \mathrm{CMH}$ environ à $20 \mathrm{~m}$ devant la paroi (piézomètres $60-3$ et 110-3, voir figure 2) et entre $-5,00$ et $-2,00 \mathrm{CMH}$ à proximité immédiate de la paroi (piézomètres 1, 7 et 8 du LCPC/CETE, voir figure 2). Derrière la paroi les piézomètres 110-1 et 60-2 montrent que la nappe est relativement stable à -3,00 CMH environ, avant d'être rabattue à partir de mi-décembre. Malheureusement, ces piézomètres ont été détruits lors des travaux de terrassement.

\section{Comparaison avec des études antérieures et perspectives}

Le suivi de cet ouvrage permet non seulement de recueillir des données indispensables pour des études numériques ou analytiques a posteriori, mais aussi pour la comparaison des résultats avec des murs de quai instrumentés antérieurement, notamment le quai d'Osaka du Port du Havre ${ }^{3}$, dont la structure est similaire à celle du quai de Port 2000, et qui a été construit dans un contexte géotechnique comparable.

L'exemple du quai d'Osaka a montré l'importance de la mise en place d'une instrumentation redondante: des défaillances de plusieurs appareillages ont sensiblement diminué la quantité de données disponibles. Pour le projet Port 2000, on n'a donc pas dispersé l'instrumentation sur plusieurs panneaux, mais on l'a mise en place sur un seul afin de recueillir des données aussi « concentrées » que possible.

En ce qui concerne le comportement de la structure durant les premières phases de travaux, l'observation du quai d'Osaka avait montré que la paroi travaille en console jusqu'après la construction de la poutre de couronnement. Ce comportement est bien confirmé par les observations faites sur le quai à Port 2000, qui ont par ailleurs permis de préciser l'ordre de grandeur des déplacements en jeu. Au quai d'Osaka, l'excavation derrière la paroi menait à un inversement du sens du déplacement, la paroi travaillant alors en profondeur toujours en console. En tête, la flexion restait toutefois positive, résultat du poids de la poutre de couronnement. Le remblaiement ne modifiait que très peu la flexion de la paroi, par contre, les déplacements négatifs vers le terrain diminuaient. Enfin le dragage conduisait à un déplacement important $(10 \mathrm{~cm})$ vers le bassin et à une décompression latérale du massif soutenu. Les observations sur la paroi de Port 2000 seront comparées aux résultats détaillés de l'étude antérieure.

\section{Références bibliographiques}

1.Port Autonome du Havre, (2003), Un nouvel espace de développement. Rapport d'Activité 2002, 48 p.

2.Rojat F, Bets N., (2004), CAPITOUL 2000, Logiciel pour l'exploitation et la représentation des mesures inclinométriques. Guide de l'utilisateur. LRPC de Toulouse $L C P C, 95$ p. - à paraître en 2004

3.Delattre L., Duca V., Mespoulhe L., (1999), Etude Expérimentale du quai Osaka du Port Autonome du Havre. Rapport interne du LCPC, 146 p. 